Published in final edited form as:

J Obstet Gynecol Neonatal Nurs. 2014 ; 43(6): 792-802. doi:10.1111/1552-6909.12505.

\title{
The Psychometric Properties of the Barkin Index of Maternal Functioning
}

\author{
Jennifer L. Barkin [assistant professor], \\ Department of Community Medicine, Mercer University, Macon, GA. \\ Katherine L. Wisner [Norman and Helen Asher Professor of Psychiatry and Behavioral \\ Sciences and Obstetrics and Gynecology], \\ Feinberg School of Medicine, Northwestern University, Chicago, IL. \\ Stephen R. Wisniewski [professor of epidemiology] \\ Graduate School of Public Health, University of Pittsburgh, Pittsburgh, PA.
}

\section{Abstract}

Objective: To evaluate the psychometric properties of the Barkin Index of Maternal Functioning (BIMF).

Design: Principal component factor rotation was used to conduct an exploratory factor analysis of the BIMF to determine if more concise versions exist.

Setting: Patients were recruited from a large, urban medical center in the Northeast.

Participants: The BIMF was administered at an initial home visit along with several other selfreport and clinical assessments to women who scored $\geq 10$ on the Edinburgh Postnatal Depression Scale during the 4- to 6-week postpartum period.

Methods: The BIMF was administered between October 2008 and September 2010. The distribution of BIMF item responses was examined along with interitem correlations. To establish construct validity, correlation coefficients were produced for the BIMF in relation to several other variables or assessments. A factor analysis was performed using principal component factor rotation.

Results: The factor analysis revealed a two-factor solution. The items that loaded on factor 1 gauged the mother's perception of her own competency in the maternal role and the items that made up factor 2 focused on the mother's needs. Items related to judgment from others and anxiety did not load on either factor.

Conclusions: The BIMF may be administered in its 18-item version, as two separate subscales, or in its original 20-item format. A clinical threshold should be developed to facilitate accurate identification of mothers who are struggling with functioning during the postpartum period.

Correspondence: Jennifer L. Barkin, PhD, Mercer University School of Medicine, Department of Community Medicine, 1550 College Street, Macon, GA 31207. barkinj@gmail.com.

The authors report no conflict of interest or relevant financial relationships. 


\section{Keywords}

postpartum functioning; maternal functioning; postpartum health; motherhood

Postpartum depression (PPD) is the most common complication of childbearing with available, proposed screening strategies (Gaynes et al., 2005). In a recent study of 10,000 mothers, 1396 (14.0\%) screened positive for PPD (Wisner et al., 2013). Despite an impressive prevalence rate of one in seven women experiencing PPD, not all are receptive to depression assessment and treatment due to the stigma associated with mental illness (Sit et al., 2009; Vesga-Lopez et al., 2008). Additionally, women often present for treatment with the goal of improved daily life function rather than to specifically reduce their depression scores as they desire more efficient performance individually and in the maternal role. These observations highlight the need for additional tools and interventions to address maternal wellness during the postpartum period. Ware, Kosinski, and Keller (1996) determined that the goal of medical care for most patients is to obtain a more effective life and to preserve functioning and well-being. More specifically, researchers have shown that new mothers are concerned with their functioning across seven domains, including (a) self-care, (b) infant care, (c) mother/child interaction, (d) psychological well-being, (e) social support, (f) management, and (g) adjustment (Barkin et al., 2010b). These researchers conducted focus groups with 31 new mothers and asked them to explain the circumstances around their low and high functioning periods. Women described peak or optimal performance in the maternal role as times where they were able to take care of themselves (self-care), take care of their infants (infant care), bond with their infants (mother/child interaction), and manage their households (management). During these times they also felt generally emotionally healthy (psychological well-being), well supported (social support), and as if they were adjusting to their added responsibilities (adjustment). Due to this interest in maternal competency and overall daily performance, it is likely that new mothers would be more receptive to assessments and, ultimately, to interventions that are focused on improved functioning during the postpartum period.

To determine if any existing maternal assessments adequately addressed maternal functioning during the 12 months following childbirth, Barkin, Wisner, Bromberger, Beach, and Wisniewski (2010a) evaluated eight of the most commonly used instruments to determine if any of the existing instruments addressed all seven domains of functioning identified in the Barkin et al. (2010b) study. Using the terms maternal instrument, maternal measure, maternal questionnaire, and maternal survey, database searches were conducted in Health and Psychosocial Instruments (1985 - 2008), CINAHL (1982 - 2008), PsycINFO (1967 - 2008), and MEDLINE (1970 - 2009). Only self-report measures and those measures intended for use during the child's first 12 months of life were considered. Each of the eight selected instruments was referenced at least 17 times in the research literature, had some published psychometric properties, and had been utilized in research studies, primary care settings, or both. The selected instruments had also been featured in systematic reviews performed by Beck (1998) and Fowles and Horowitz (2006). The eight selected measures included the following: (a) the Gratification Checklist (Mercer, 2004; Russell, 1974), (b) the Infant Care Survey (Froman \& Owen, 1989), (c) How I Feel About My Baby Now Scale 
(Leifer, 1977), (d) Parenting Sense of Competence Scale (PSOC) (Gibaud-Wallston \& Wandersmann, 1978), (e) Myself as Mother Scale (Walker, Crain, \& Thompson, 1986), (f) My Baby Scale (Walker et al., 1986), (g) What Being the Parent of a New Baby is LikeRevised (WPL-R) (Pridham \& Chang, 1989), and (h) Inventory of Functional Status After Childbirth (IFSAC) (Fawcett, Tulman, \& Myers, 1988). After evaluation of these tools by these authors, it was found that none of the selected instruments addressed all seven components of postpartum functioning.

When comparing the instruments, the IFSAC provided the most comprehensive coverage of the reviewed instruments, addressing six of the seven domains to varying degrees. This result was as expected; the IFSAC is the only selected instrument that was designed specifically for assessment of postpartum maternal functioning. The mother's psychological well-being and feelings about her life postchildbirth are not included as part of the IFSAC. Additionally, the IFSAC focuses on resumption of woman's pre-birth activities and does not adjust for the natural reprioritization that takes place as the result of having a child. The results of this study confirmed the need for a new, more inclusive tool for the assessment of postpartum functional status.

The Barkin Index of Maternal Functioning (BIMF) (Barkin et al., 2010b) was created to address the aforementioned need by employing a comprehensive, patient-centered approach to development. The goal of the development process was to design a measure that addressed all seven domains of maternal functioning, was viable in research and clinical settings, and had favorable psychometric properties. Initial item development for the BIMF was based on the qualitative data derived from three focus groups of new mothers $(N=31)$. Because the 20-item BIMF was based on the experiences of women experiencing new motherhood, it is inherently patient centered.

The BIMF was included as one of the assessment tools used in the Identification and Therapy of Postpartum Depression Study (Wisner et al., 2013) and was analyzed in a subset of participants for internal reliability and construct validity. Content validity had already been ensured by using qualitative data from new mothers to inform initial item development. The initial psychometric analysis revealed a Cronbach's alpha of 0.87 and adequate construct validity was observed (Barkin et al., 2010b). The initial psychometric properties were promising, and the BIMF has been requested by various individuals or groups who are interested in a patient-centered alternative or complement to depression screening. These groups of providers comprise pediatricians who have frequent access to new mothers via well-child visits, obstetricians, organizations with home visiting programs, and nurse researchers. All these professionals are interested in capturing maternal functional status as a primary maternal health outcome. Due to the level of interest in the BIMF as a clinical tool and an outcomes measure, the developers deemed a broader psychometric evaluation a necessary next step. In 2011, 346 completed baseline assessments from postpartum women were available for an extensive examination of the BIMF's psychometric properties. The following research questions are the focus of this psychometric evaluation:

1. How did women respond to the BIMF's original 20 items and how are the items related to each other? 
2. What is the reliability and factor structure of the 20 -item BIMF in this sample of 346 women?

3. Does the factor analysis support the 20-item version of the BIMF or does it identify other versions that may be more concise?

4. If other BIMF versions are identified by the aforementioned factor analysis, do they possess adequate psychometric properties?

5. If other BIMF versions are identified, are there still instances in which the original BIMF may still be appropriate for use?

\section{Methods}

\section{Study Population}

The Identification and Therapy of Postpartum Depression Study represents a comprehensive screening strategy designed to identify women with PPD (Wisner et al., 2013). The BIMF was administered between October 2008 and September 2010. All pregnant women delivering at a large tertiary hospital in the Northeast United States were offered inclusion into the study. During the hospitalization for birth, women were asked if they would accept screening for postpartum depression by phone by the research team at 4-to 6-weeks postpartum. Those women who had positive screens on the Edinburgh Postnatal Depression Scale (EPDS) (Cox, Holden, \& Sagovsky, 1987) defined as a score $\geq 10$, were offered a home (baseline) visit to conduct a diagnostic evaluation with the Structured Clinical Interview for DSM-IV (SCID).

\section{Assessments}

In addition to the SCID and the BIMF, several other self-report and clinical interview measures were administered at the home visit. The 20-item $\mathrm{BIMF}\left(\mathrm{BIMF}_{20}\right)$ has a possible range of 0 to 120 with a total score of 120 indicating optimal maternal functioning. Selfreport measures included the Gratification Checklist (GRAT), which assesses gratification in the maternal role, and the 12-item Short-Form Health Survey (SF-12) (Ware et al., 1996), a measure of general health-related functioning. Clinical interviews included the Structured Interview Guide for the Hamilton Depression Rating Scale with Atypical Depression Supplement (SIGH-ADS) (Williams \& Terman, 2003), the Global Assessment Scale (GAS) (Endicott, Spitzer, Fleiss, \& Cohen, 1976), and the SCID (First, 2005) that was used to determine current or lifetime psychiatric diagnoses. Demographic characteristics including race, ethnicity, age (of mother and infant), mother's education level, type of health insurance, and marital status were collected at the screening call preceding the home visit. These variables were used to describe the study sample (Table 1) and to examine concurrent and discriminant validity (Table 6).

\section{Analytic Methods}

The 346 study participants completed all 20 of the BIMF items. Percentages were used to describe the study sample characteristics for categorical variables. Means, standard deviations, medians, and ranges were obtained to describe continuous characteristics. 
Percentages were also provided to reflect the distribution of endorsed item responses which range from strongly disagree to strongly agree. Interitem correlations for the $\mathrm{BIMF}_{20}$ are presented in the form of Spearman rank correlation coefficients. Principal component factor rotation was used to conduct the exploratory factor analysis. Spearman correlation coefficients were generated to assess the relationship between the resultant 18-item BIMF's $\left(\mathrm{BIMF}_{18}\right)$ total score and the subscale (factor) scores. Evidence of discriminant and concurrent validity for each of the BIMF versions was also expressed in terms of Pearson correlation coefficients. Specifically, correlations between the total score for each version of the BIMF and measures of global functioning, depression, maternal gratification, and mother and infant age were generated.

\section{Participant Characteristics}

The characteristics of the study sample are displayed in Table 1 . The majority of the sample was White (68\%), unmarried (56\%), and utilizing public health insurance (53\%). On average, the mothers were age 28.2 years and infants were age 6.2 weeks. The average $(S D)$ SIGH-ADS-29 score was 22 (6.3), which is indicative of depression of moderate severity. On average, women had a BIMF average $(S D)$ total score of 79.6 (16.2).

\section{Results}

\section{Distribution of Responses to Items}

Table 2 provides the distribution of responses for each of the BIMF's 20 items. Several of the items related to the mother's needs (Item 2 - "I feel rested," Item 8 - "I am getting enough adult interaction," and Item 11 - "I take a little time each week to do something for myself") were left skewed indicating that negative responses were endorsed more frequently for these particular items. Conversely, items related to the mother's evaluation of her competence in the maternal role (Item 1 - "I am a good mother," Item 19 - "As time goes on I am getting better at taking care of my baby," and Item 20 - "I am satisfied with the job I am doing as a new mother") tended to be positively skewed. Items 16 ("I worry about how other people judge me as a mother") and 18 ("Anxiety or worry often interferes with my mothering ability") were relatively evenly distributed across the response categories relative to the other BIMF items.

\section{Item-item correlations}

The interitem correlations for the $\mathrm{BIMF}_{20}$ are displayed in Table 3. Item 19 ("getting better at taking care of my baby") and Item 20 ("satisfied with the job I'm doing") were strongly correlated with each other $(r=.70)$ and had correlations over .50 with Items 1 (good mother), 10 (trust instincts), and 14 (good decisions). Good decision making (Item 14) was related to trusting instincts (Item 10) $(r=.56)$ and taking good care of baby's physical needs (Item 12) $(r=.53)$. The items related to child care help (Items 6 and 7) were correlated ( $r$ $=.66$ ) with each other. Mom and baby getting into a routine (Item 15) and satisfaction (Item $20)$ were also related $(r=.52)$. Interactional Items 4 and 5 were associated $(r=.54)$. 


\section{Exploratory Factor Analyses}

The results of the exploratory factor analysis are displayed in Table 4. The scree plot of eigenvalues and the proportion of variance explained were examined to determine that two oblique factors should be retained. In terms of the variance, $70.72 \%$ of the variance was explained by Factor 1 and 18.80 was explained by Factor 2. Items with loadings $\geq .40$ were considered to load on a given factor. Within the two-factor solution for the $\mathrm{BIMF}_{20}$, Items 16 and $18 \mathrm{did}$ not load on either factor. Therefore, both of these items were excluded from subsequent analyses. None of the items cross-loaded on both factors. The items that loaded on Factor 1 (Mom's Competency) gauged the mother's perception of her own competency in the maternal role and the items that made up Factor 2 (Mom's Needs) were focused on the mother's needs. The following lists provide the items that loaded on Factor 1 and 2.

Factor 1 or the Mom's Competency Scale included (a) I am a good mother, (b) I am comfortable with the way I've chosen to feed my baby, (c) My baby and I understand each other, (d) I am able to relax and enjoy time with my baby, (e) I trust my instincts when it comes to taking care of my baby, (f) I am taking good care of my baby's physical needs, (g) I make good decisions about my baby's health and well-being, (h) My baby and I are getting into a routine, (i) I am able to take care of my baby and my other responsibilities, (j) As time goes on, I am getting better at taking care of my baby, and (k) I am satisfied with the job I am doing as a new mother. Factor 2 or the Mom's Needs Scale included (a) I feel rested, (b) There are people in my life that I can trust to care for my baby, (c) I am comfortable allowing a trusted friend or relative to care for my baby, (d) I am getting enough adult interaction, (e) I am getting enough encouragement from other people, (f) I take a little time each week to do something for myself, and (g) I am taking good care of my physical needs.

\section{Reliability}

The Cronbach's alpha for the 20-item and 18-item BIMFs were .87 and .88, respectively. The Cronbach's alpha for the Mom's Competency and Mom's Needs Scales were .88 and .77 , respectively.

\section{Descriptive Statistics and Interscale Correlations}

Means, standard deviations, and ranges are displayed in Table 5 for each of the factors (scales) as well as for the total 18-item BIMF. Factors 1 and 2 are correlated with each other $(r=.44)$ as with as with the 18 -item BIMF total score ( $r=.88$ and .80 , respectively).

\section{Validity}

Concurrent and discriminant validity were examined for the BIMF20, the BIMF18, and the Mom's Competency and Mom's Needs subscales. The results are displayed in Table 6. Maternal gratification was significantly and positively correlated with each of the BIMF total scores and each of the subscale scores, as was mental functioning. This result suggests that as the levels of maternal functioning increases, so do the levels of maternal gratification and mental functioning. Physical functioning was not significantly associated with maternal functioning in any case. As expected, depression was inversely related to maternal functioning and the relationship was significant in each case, with the exception of the Mom's Competency Scale. Infant age at baseline was not related to maternal functioning. 
The restricted observed range ( $4-11$ weeks) for infant age at the home visit may have influenced this lack of finding. The mother's age had an inverse, significant relationship with the $\mathrm{BIMF}_{20}$, the $\mathrm{BIMF}_{18}$, and the Mom's Competency Scale. This finding implies diminished self-reported maternal functioning in older mothers. The mother's age and the score on the Mom's Needs subscale was not significantly associated.

\section{Discussion}

In summary, the BIMF may be administered in its 18 -item version as two separate subscales or in its original 20-item format. An exploratory factor analysis of the BIMF resulted in a two-factor solution including 18 of the 20 items. Items focused on judgment from others (Item 16) and anxiety (Item 18) did not load on either factor and were excluded from the $\mathrm{BIMF}_{18}$. Although these two items did not load on either factor, the $\mathrm{BIMF}_{20}$ and $\mathrm{BIMF}_{18}$ are comparable in terms of internal reliability and construct validity. If a researcher or health care provider is interested in women's responses to questions related to judgment and anxiety in new motherhood, that researcher or health care provider may opt for the $\mathrm{BIMF}_{20}$. Conversely, if they prefer a measure in which all items loaded cleanly on an identified factor, they may choose to administer the $\mathrm{BIMF}_{18}$. The results of a subsequent factor analysis of the BIMF $_{18}$ met the following key interpretability criteria: (a) At least three items had significant loadings on each factor, (b) the items that loaded on each retained factor shared conceptual meaning, (c) the items that loaded on the two different factors measured different constructs, and (d) the rotated factor pattern demonstrated a simple structure (Hatcher, 1994). The $\mathrm{BIMF}_{18}$ also displayed adequate internal consistency (Cronbach's alpha $=.88$ ) and construct validity as the $\mathrm{BIMF}_{18}$ was significantly correlated with maternal gratification, depression, mental functioning, and global functioning. In each of these cases, the direction and significance of the relationship observed was as expected. The lack of association between maternal functioning $\left(\mathrm{BIMF}_{18}\right)$ and infant age may be explained by the restricted range of infant age at the home visit as it is possible that maternal functioning does not change significantly in the 7-week window between 4- and 11-weeks postpartum.

Two subscales of the BIMF 18 were identified (a) Mom's Competency and (b) Mom's Needs Scales. Both scales have adequate internal consistency and are also strongly (>.40), positively, and significantly correlated with maternal gratification. The ability to measure the level to which the mother's needs are being satisfied is particularly advantageous, as women have described a challenge in managing infant and self-care concurrently (Barkin \& Wisner, 2013). Several studies have reported that maternal self-care that has conceptual overlap with the BIMF's Mom's Needs Scale, tends to lag behind other areas of maternal role functioning in the postpartum period (McVeigh, 1998; Posmontier, 2008; Tulman, Fawcett, Groblewski, \& Silverman, 1990). The left skewed distributions for Items 2, 8, and 11 in Table 2 support this position.

Prior reports of the BIMF included the evaluation of reliability and construct validity in a subsample of the participants reported in the current analyses and are consistent with these results. With the addition of this factor analysis, evidence to support favorable psychometrics for the BIMF is approaching comprehensive. In describing the development process for the IFSAC, Fawcett et al. (1988) provided evidence of content validity, test- 
retest reliability, inter-rater reliability, and internal consistency reliability. In a review of IFSAC-related literature, Aktan (2007) noted that criterion-related validity has not been explored and methods of assessing the concurrent validity of the IFSAC are suggested. Subsequently, researchers have examined the relationship between the IFSAC and other variables (Ahn \& Youngblut, 2007). However, it is unclear whether the researchers intended to test for the validity of the IFSAC, and conclusions are not framed in terms of validity testing. In the original development article, the Cronbach's alpha for internal consistency reliability was .76 for the whole IFSAC scale. The subscale Cronbach's alpha were as follows (a) self-care .56, (b) household .64, (c) social and community .67, (d) infant care .92, (e) and occupational .98 . The originally reported Cronbach's alpha for the self-care, household, and social and community subscales are not ideal and indicate the need for modification. In a study of the reliability and validity of the IFSAC that included 191 Turkish women (Ozkan \& Umran, 2007), the authors found Cronbach's alpha less than .70 for all subscales, with the exception of household activities (.83). The self-care subscale was again problematic with an alpha of .53. More recently, researchers have shown an improvement in internal consistency reliability of the total IFSAC scale. In a study of predictors of postpartum health status in the first 3 months after childbirth, Ahn and Youngblut (2007) reported Cronbach's alpha for the IFSAC of .98 at Time 1 and .94 at Time 2. In a study comparing functional outcomes in mothers with and without postpartum depression, Postmontier (2008) reported an alpha reliability of .85 for the overall IFSAC scale. To the authors' knowledge, a factor analysis of the IFSAC has not been reported in the literature; a factor analysis would serve to identify unique subscales or confirm the existing IFSAC subscales.

The strengths of this investigation include the use of a sociodemographically diverse sample from a large urban hospital and the patient-centered approach to the development of the measure itself. Patient-centered outcomes are, by definition, developed to assess the patient's perspectives, interests, and values throughout the research process and are intended to be practically relevant. The primary weakness of this evaluation is that it was focused on a single sample of individuals who all screened positive for PPD.

\section{Implications for Practice}

Currently, the $\mathrm{BIMF}_{20}$ is scored on a continuous scale from 0 to 120 ; a score of 120 indicates optimal functioning. However, a numeric threshold that indicates in need of functional intervention should be identified. Such a threshold would facilitate accurate and timely identification of struggling mothers by health care professionals. The BIMF could be used in tandem with PPD screening, allowing for a more comprehensive assessment of maternal wellness. The $\mathrm{BIMF}_{20}$ takes approximately 5 minutes to complete and has been easily administered in a variety of settings including hospitals, women's behavioral health clinics, and through home visiting programs.

Due to the fact that specific interventions have not yet been developed to support functional evaluation, the identification of a woman in need of intervention could be followed by a discussion with the treating physician, a nurse, or by a referral to a mental health professional. If a woman was willing, the health care provider could discuss the results of 
the BIMF screening and areas for potential improvement. For example, if a woman indicated that anxiety or worry often interfered with her mothering ability (Item 18), this could be used as a springboard for discussion. As mentioned, the psychometric evaluation also supports the use of the BIMF as two separate subscales (Mom's Needs, Mom's Competency). For example, the Mom's Needs Scale is brief and would allow a clinician or researcher to quickly assess the level to which the mother's needs were fulfilled. This issue of maternal self-care is important as women often neglect their own physical and emotional needs in the chaos of motherhood (Barkin \& Wisner, 2013). The Mom's Needs Scale provides an alternative to the IFSAC self-care subscale, which has been defined as problematic (McVeigh \& Chaboyer, 2002). The purpose of assessment, target population, and administrator preference should determine which BIMF version is used.

\section{Conclusions}

In summary, the BIMF addresses all seven domains of maternal functioning as identified in a qualitative study of new mothers and has thus far been well received by patients and research subjects (Barkin et al., 2010b). Evidence of discriminant and concurrent validity has been reported for all versions, and the BIMF is supported by the factor analysis presently reported. Future studies should evaluate the BIMF in other relevant populations including one whose members have not screened positive for depression. The examination of change in maternal function across the first-year postbirth would also add to the evaluation of the BIMF. Additionally, interventions aimed at improved functioning should be developed for mothers who exhibit poor functioning.

\section{Acknowledgement}

Supported by an internal development fund at the University of Pittsburgh and the Identification and Therapy of Postpartum Depression Study (R01 MH071825).

\section{REFERENCES}

Ahn S, \& Youngblut JM (2007). Predictors of women's postpartum health status in the first 3 months after childbirth. Asian Nursing Research, 1(2), 136-146. [PubMed: 25030750]

Aktan NM (2007). Functional status after childbirth. Clinical NursingResearch, 16(3), 195-211.

Barkin JL, Wisner KL, Bromberger JT, Beach SR, \& Wisniewski SR (2010a). Assessment of functioning in new mothers. Journal of Women's Health, 19(8), 1493-1499.

Barkin JL, Wisner KL, Bromberger JT, Beach SR, Terry MA, \& Wisniewski SR (2010b). Development of the Barkin Index of Maternal Functioning. Journal of Women's Health, 19(12), 2239-2246.

Barkin JL, \& Wisner KL (2013). The role of maternal self-care in new motherhood. Midwifery, 29(9), 1050-1055. [PubMed: 23415369]

Beck CT (1998). A review of research instruments for use during the postpartum period. Journal of Maternal/Child Nursing, 23(5), 254-261.

Cox JL, Holden JM, \& Sagovsky R (1987). Detection of postnatal depression. Development of the 10item Edinburgh Postnatal Depression Scale. British Journal of Psychiatry, 150(6), 782-786. [PubMed: 3651732]

Endicott J, Spitzer RL, Fleiss JL, \& Cohen J (1976). The Global Assessment Scale: A procedure for measuring overall severity of psychiatric disturbance. Archives of General Psychiatry, 33(6), 766771. [PubMed: 938196]

Fawcett J, Tulman L, \& Myers ST (1988). Development of the inventory of functional status after childbirth. Journal of Nurse-Midwifery, 33(6), 252-260. [PubMed: 3236074] 
First MB (2005). Structured clinical interview for DSM-IV-TR Axis I disorders: Patient edition. New York, NY: Columbia University.

Fowles ER, \& Horowitz JA (2006). Clinical assessment of mothering during infancy. Journal of Obstetric, Gynecologic, \& Neonatal Nursing, 35(5), 662-670.

Froman RD, \& Owen SV (1989). Infant care self-efficacy. Research and Theory for Nursing Practice, 3(3), 199-211.

Gaynes BN, Gavin N, Meltzer-Brody S, Lohr KN, Swinson T, Gartlehner G, ... Miller WC (2005). Perinatal depression: Prevalence, screening accuracy, and screening outcomes. Evidence Report/ Technology Assessment, 119, 1-8.

Gibaud-Wallston J, \& Wandersmann LP (1978). Development and utility of the parenting sense of competence scale. Washington, DC: John F. Kennedy Center for Research on Education and Human Development.

Hatcher L (1994). A step-by-step approach to using the SAS system for factor analysis and structural equation modeling. Cary, NC: SAS Institute.

Leifer M (1977). Psychological changes accompanying pregnancy and motherhood. Genetic Psychology Monographs, 95, 55-96. [PubMed: 849833]

McVeigh C (1998). Functional status after childbirth in an Australian sample. Journal of Obstetric, Gynecologic, \& Neonatal Nursing, 27(4), 402-409.

McVeigh C, \& Chaboyer W (2002). Reliability and validity of the IFSAC when used in an Australian population. Nursing \& Health Sciences, 4(3), 107-112. [PubMed: 12153408]

Mercer RT (2004). Becoming a mother versus maternal role attainment. Journal of Nursing Scholarship, 36(3), 226-232. [PubMed: 15495491]

Ozkan S, \& Umran S (2007). The study of validity and reliability of inventory of functional status after childbirth. TAF Preventive Medicine Bulletin, 6(3), 199-208.

Posmontier B (2008). Functional status outcomes in mothers with and without postpartum depression. Journal of Midwifery and Women's Health, 53(4), 310-318.

Pridham KF, \& Chang AS (1989). What being the parent of a new baby is like: revision of an instrument. Research in Nursing \& Health, 12(5), 323-329. [PubMed: 2798953]

Russell CS (1974). Transition to parenthood: Problems and gratifications. Journal of Marriage and the Family, 36(2), 294-302.

Sit DK, Flint C, Svidergol D, White J, Wimer M, Bish B, \& Wisner KL (2009). Best practices: an emerging best practice model for perinatal depression care. Psychiatric Services, 60(11), 14291431. [PubMed: 19880455]

Tulman L, Fawcett J, Groblewski L, \& Silverman L (1990). Changes in functional status after childbirth. Nursing Research, 39(2), 70-75. [PubMed: 2315069]

Vesga-Lopez O, Blanco C, Keyes K, Olfson M, Grant BF, \& Hasin DS (2008). Psychiatric disorders in pregnant and postpartum women in the United States. Archives of General Psychiatry, 65(7), 805815. [PubMed: 18606953]

Walker LO, Crain H, \& Thompson E (1986). Maternal role attainment and identity in the postpartum period: Stability and change. Nursing Research, 35(2), 68-71. [PubMed: 3633507]

Ware JE Jr., Kosinski M, \& Keller SD (1996). A 12-item short-form health survey: Construction of scales and preliminary tests of reliability and validity. Medical Care, 34(3), 220-233. [PubMed: 8628042]

Williams J, \& Terman M (2003). Structured interview guide for the Hamilton Depression Rating Scale with atypical depression supplement (SIGH-ADS). New York, NY: State Psychiatric Institute.

Wisner KL, Sit DK, McShea MC, Rizzo DM, Zoretich RA, Hughes CL, \& Hanusa BH (2013). Onset timing, thoughts of self-harm, and diagnoses in postpartum women with screen-positive depression findings. JAMA Psychiatry, 70(5), 490-498. [PubMed: 23487258] 


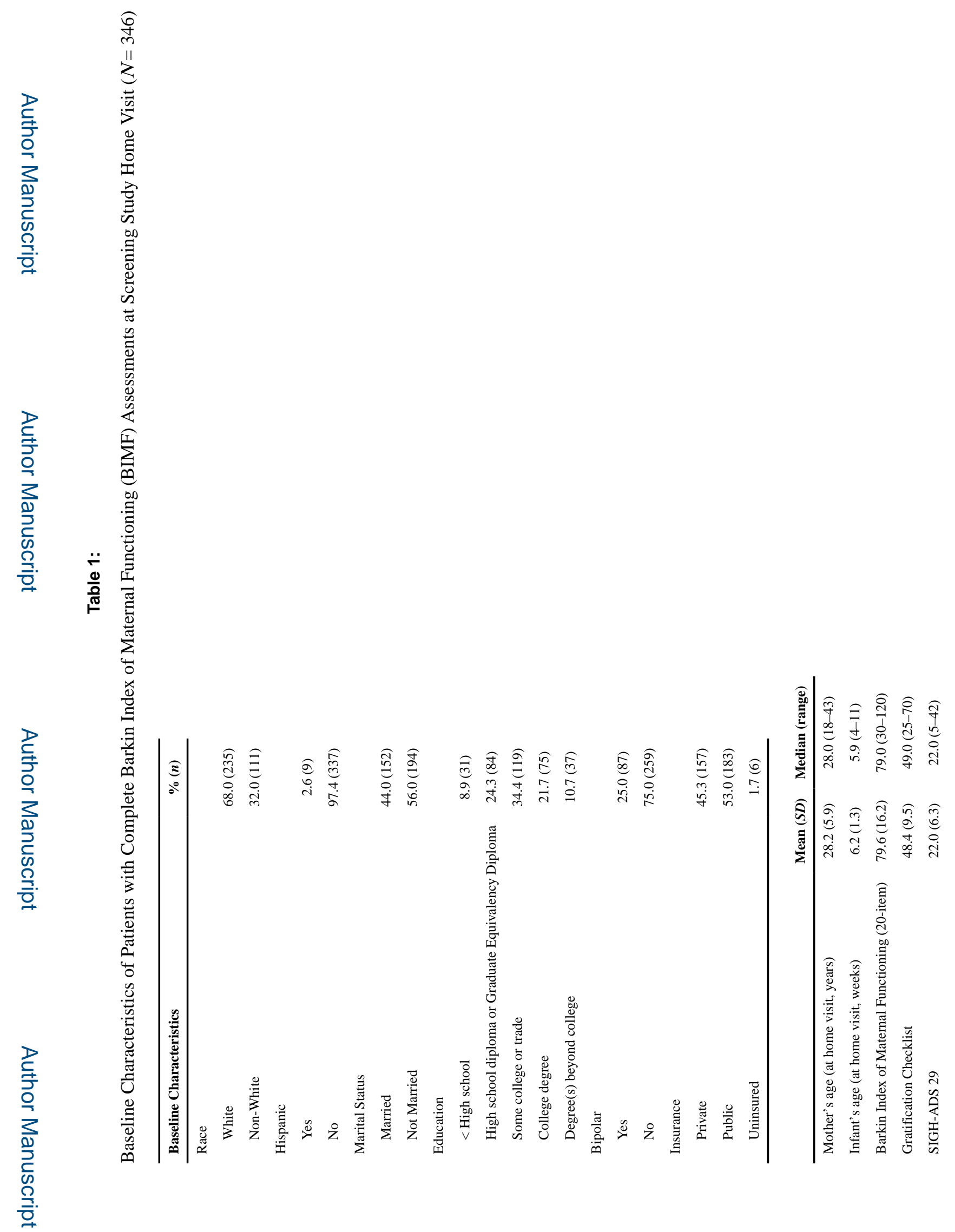




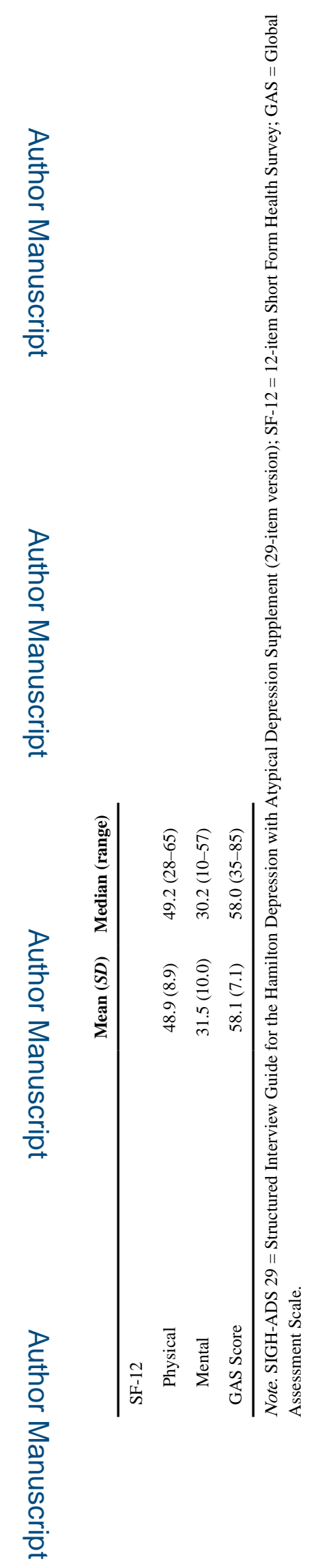


Barkin et al. 


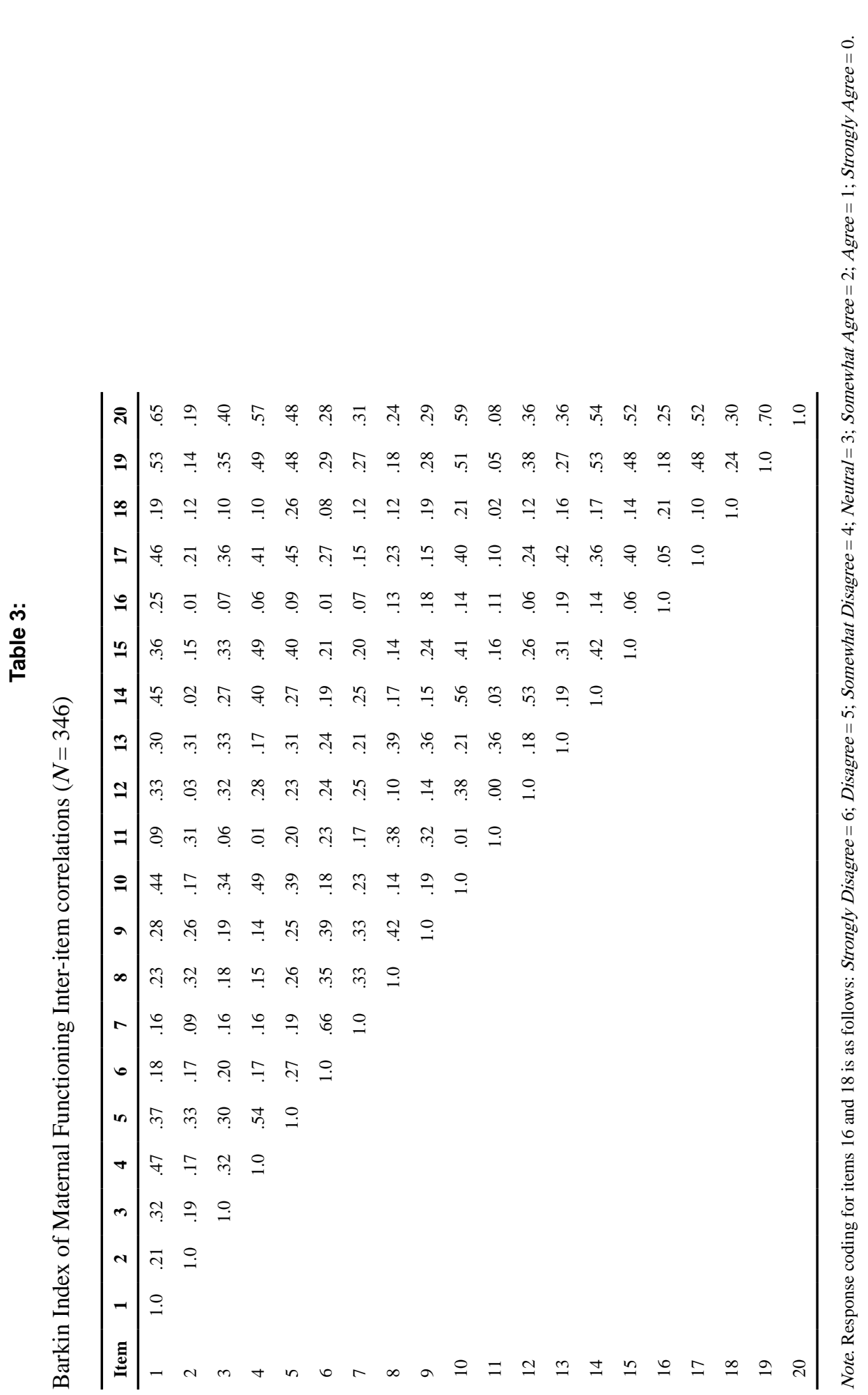


Barkin et al.

\section{을 \\ }

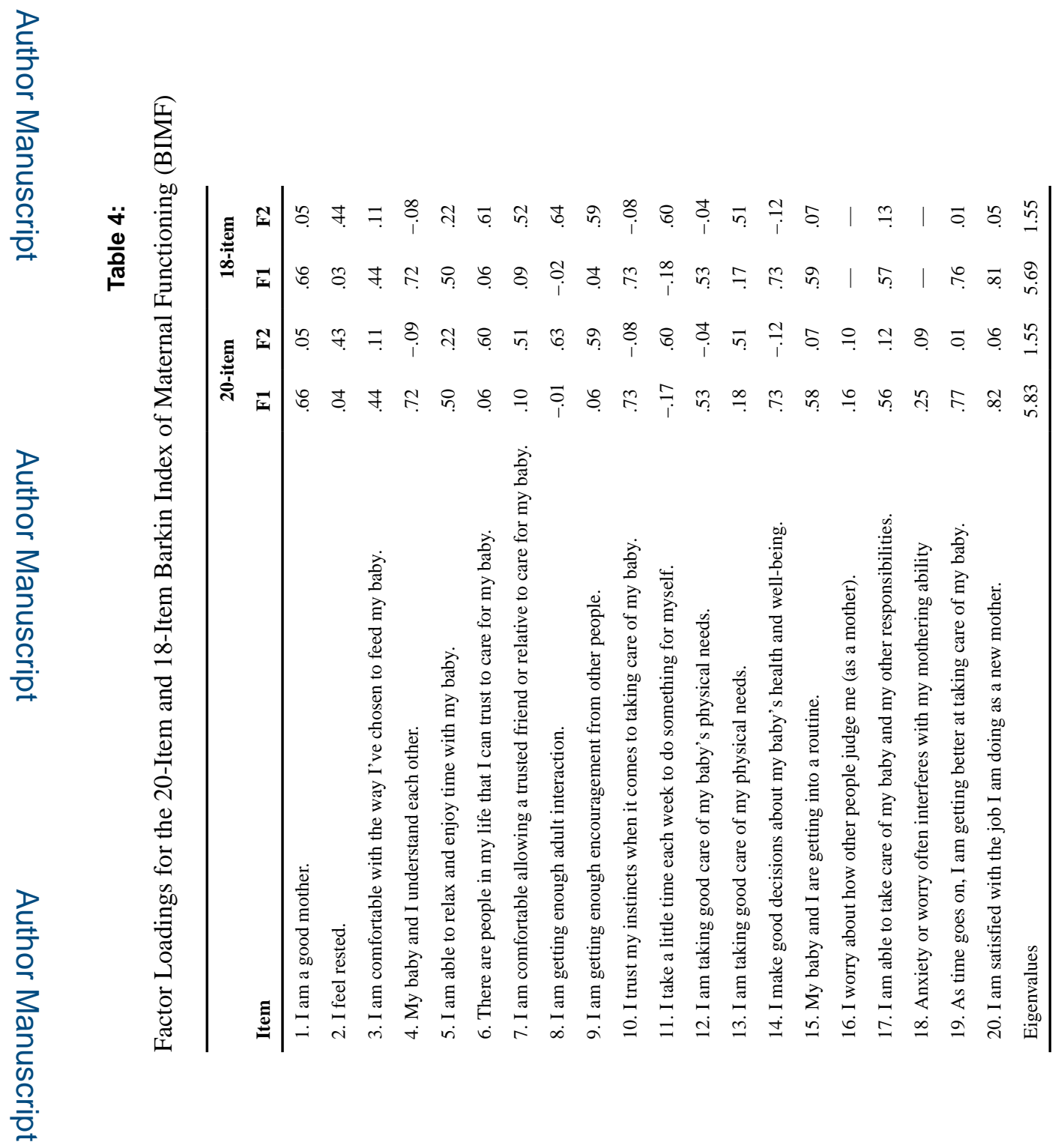

J Obstet Gynecol Neonatal Nurs. Author manuscript; available in PMC 2020 March 20. 

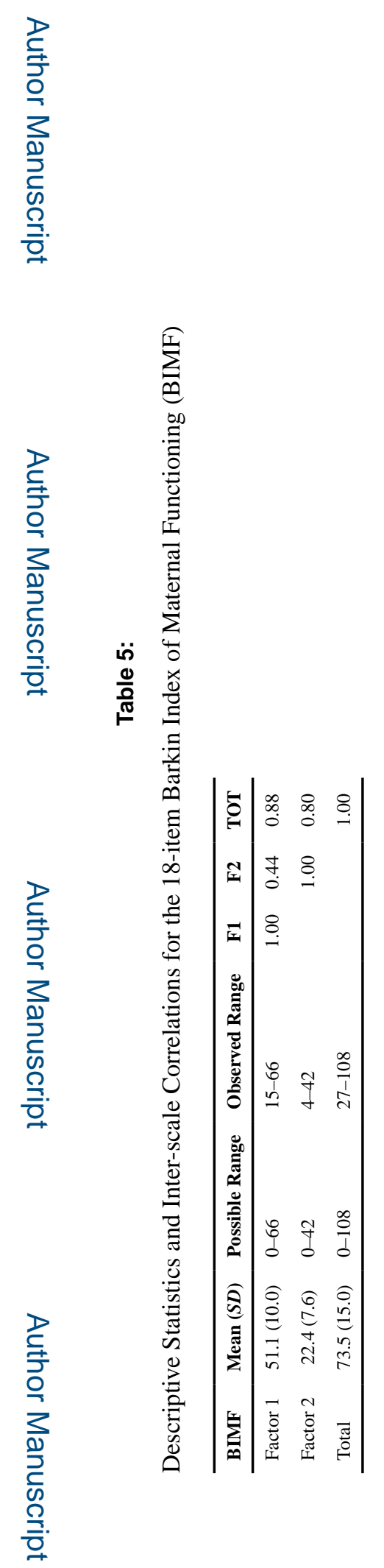

J Obstet Gynecol Neonatal Nurs. Author manuscript; available in PMC 2020 March 20. 


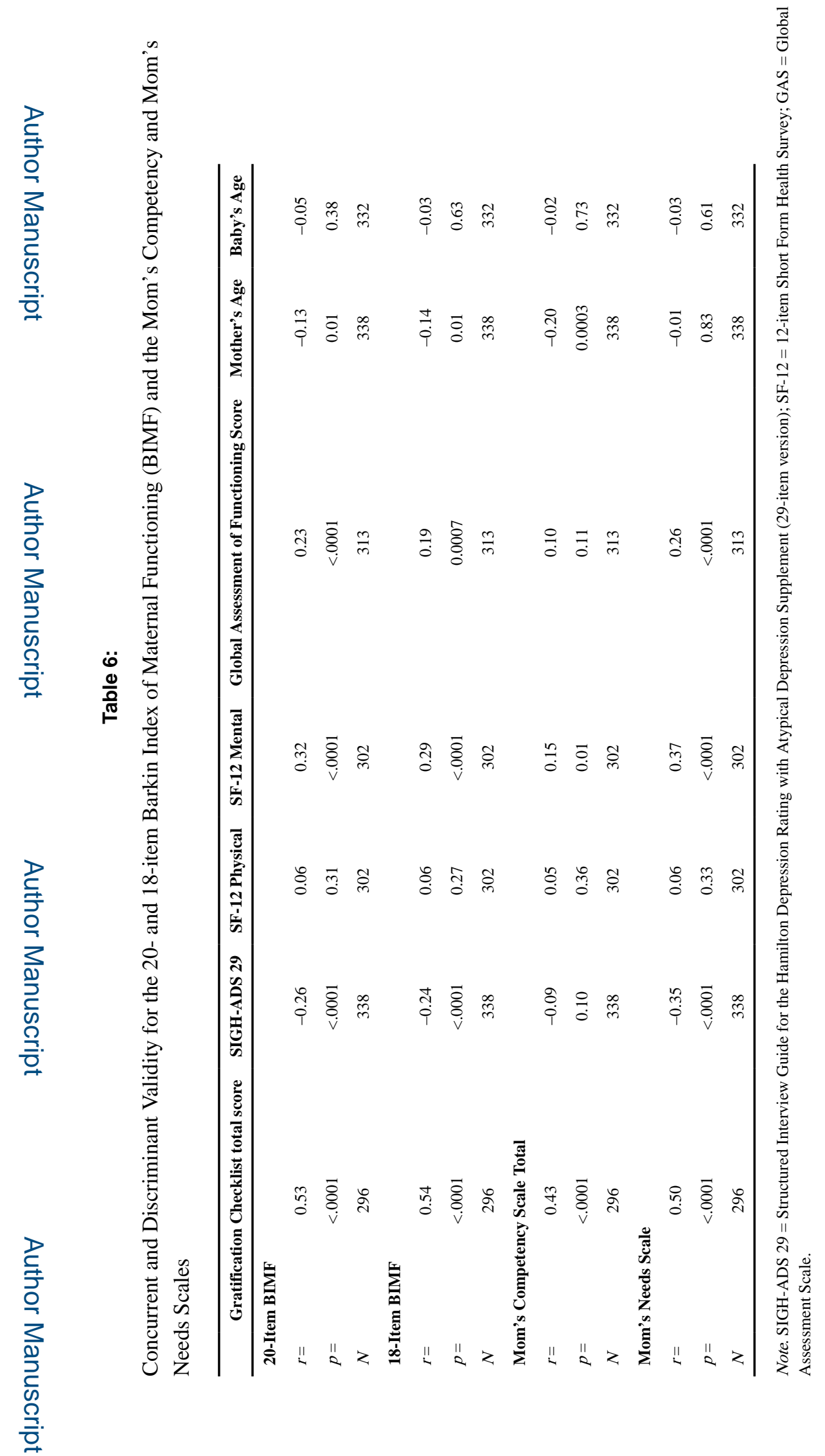

J Obstet Gynecol Neonatal Nurs. Author manuscript; available in PMC 2020 March 20. 